\title{
A Computerized Cognitive Training protocol's impact on the cognitive functioning of elderly people with dementia.
}

\author{
Ana Antunes, Carla Pombo, Cátia Gameiro \\ Unidade de Neuroestimulação na Demência - Casa de Saúde da Idanha - IIHSCJ
}

\section{Objective}

The aim of this study is to evaluate the impact of a computerized cognitive training protocol on the cognitive functioning of elderly people with dementia.

\section{Methods}

This is a descriptive study of the impact of a computerized cognitive training protocol on 6 domains of cognitive functioning in a sample of 50 elderly people with dementia.

The protocol refers to a set of computerized cognitive training activities selected according to the primary target areas of cognitive rehabilitation in dementia (viso-constructive, language, executive functions, calculus, memory and attention).

The elderly people completed a total of 15 sessions of computerized training with periodicity of 3 times a week.

\section{Results}

The main results show that approximately $80 \%$ of the sample presented levels of improvement on 3 of the 6 cognitive domains of the protocol, being that $100 \%$ of the sample presented values of improvement in, at least, one domain of the program's main focus mental functions.

Both domains "attention" and "memory" represent the cognitive functions with the best value of improvement $(90 \%$ and $86.7 \%$, respectively).

The "viso-constructive ability" domain is the cognitive function with the lower value of improvement (10\%).

Number of Domains with Improvement

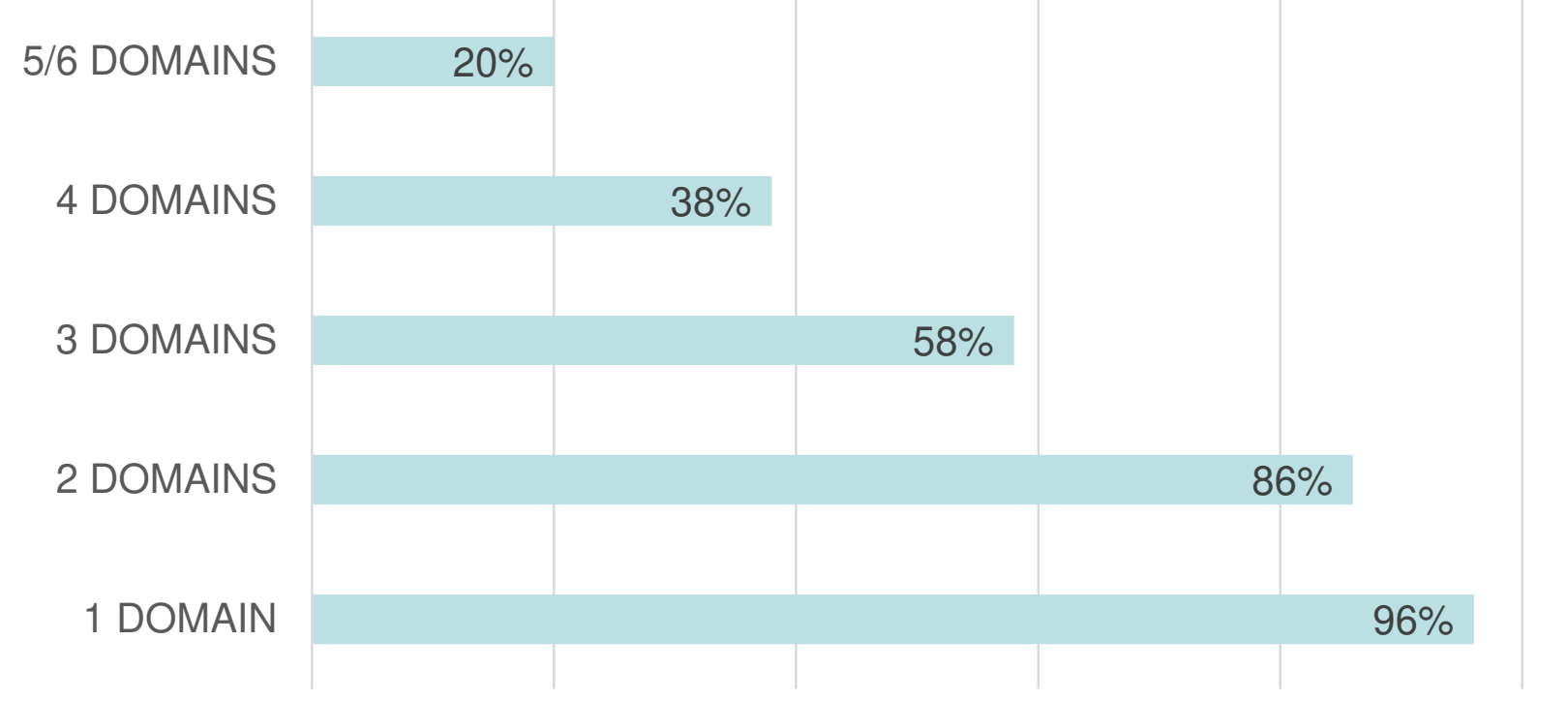

\section{Conclusion}

The entire sample presented cognitive improvement values after the implementation of the 15 sessions of the cognitive training, which seems to demonstrate the importance of this nonpharmacological therapy on the multidimensional approach in dementia.

Results suggest that both cognitive domains "attention" and "memory" should be privileged in the planning of cognitive training sessions, as they have emerged as the domains with the best value of improvement and are the primary areas on autonomy maintenance.

The low results of the "viso-constructive" domain in terms of cognitive improvement value should be aim of discussion of its inclusion on exercises of computerized training protocol.

The results obtained from the implementation of the intervention plan point us to the importance of the individualized approach in the planning, intervention and rehabilitation process of people with dementia. Activities with rehabilitation intentions, based on new technologies, have proved to be more motivating for the elderly people, and have shown a significant rehabilitation impact when compared to traditional ones.
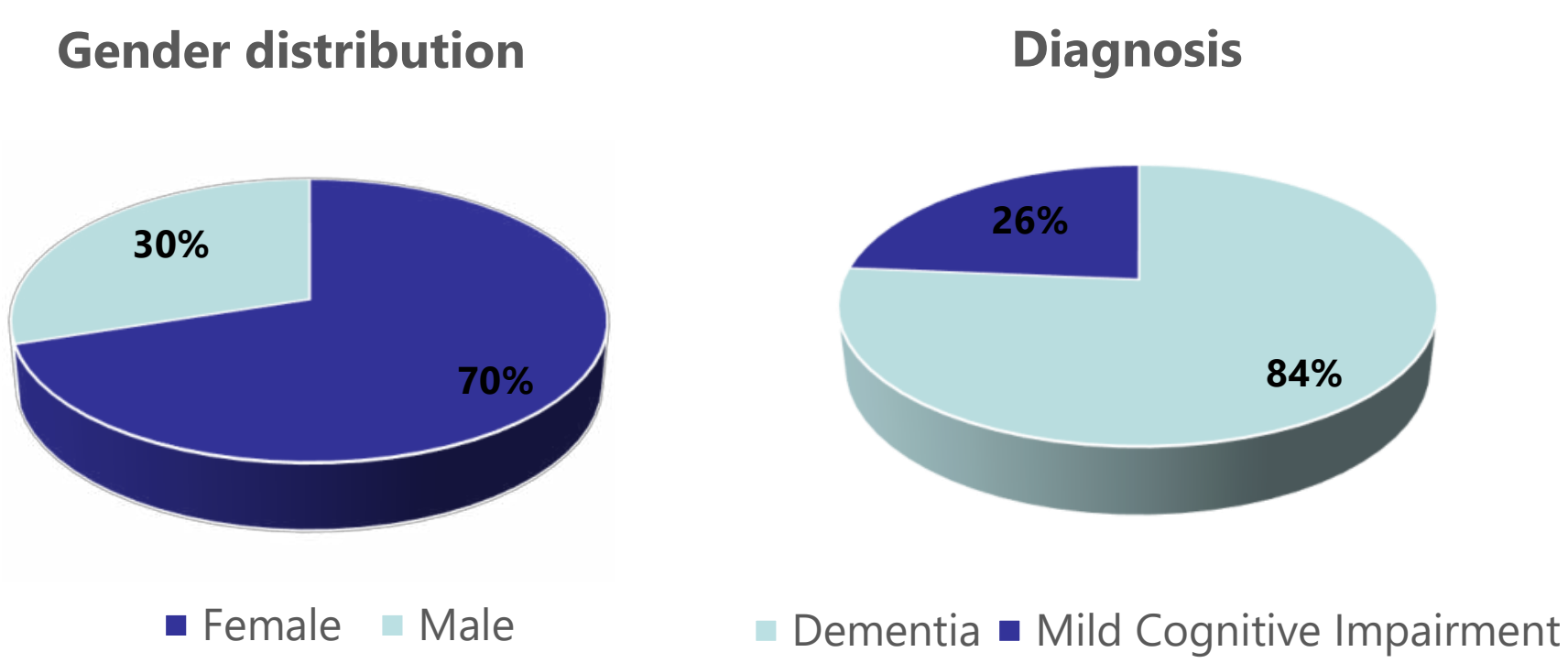

Sample's percentage with improvement per cognitive domain

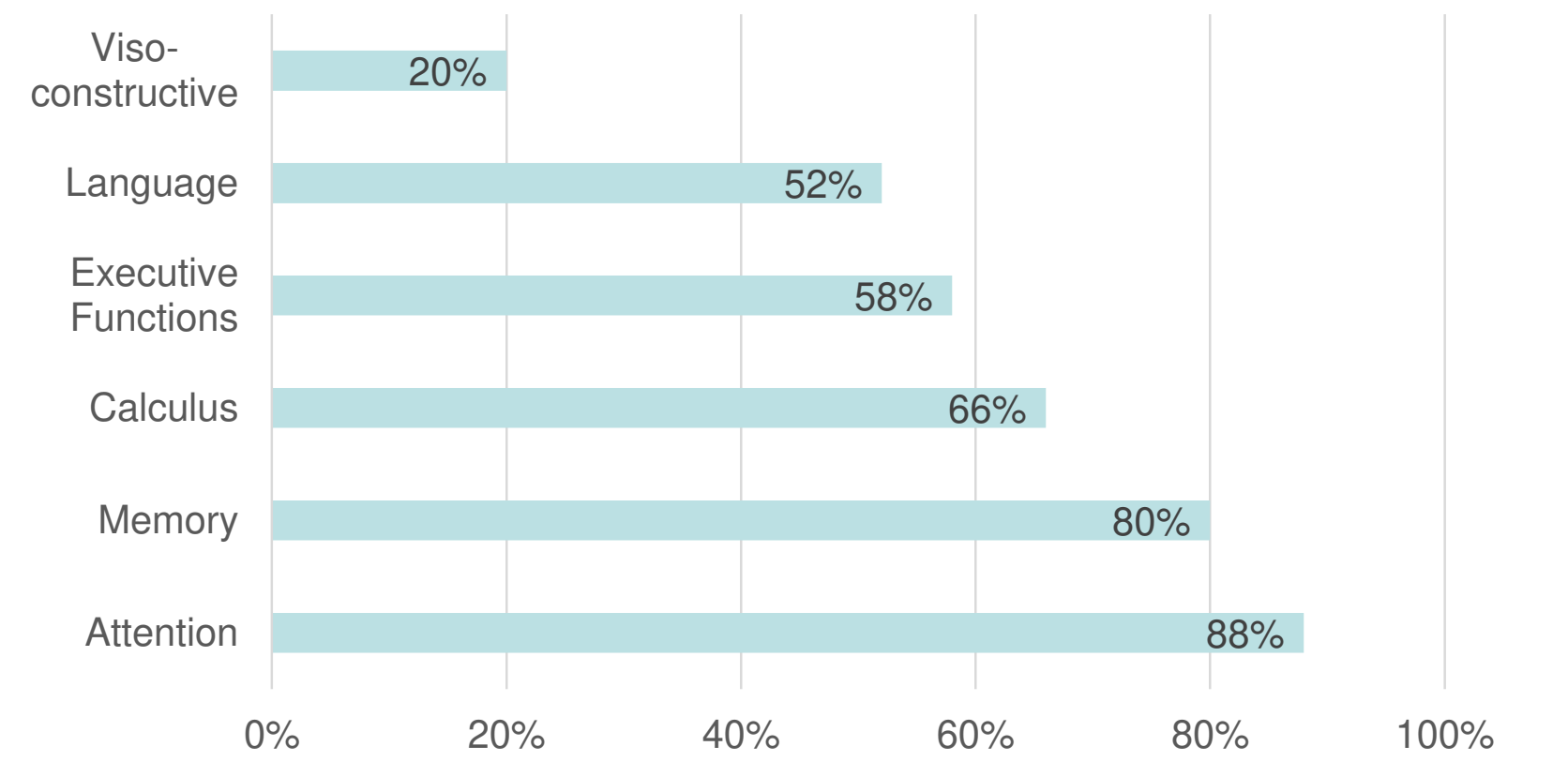

Copyright (c) 2019 Ana Antunes, Carla Pombo, Cátia Gameiro Unidade de Neuroestimulação - IISCJ - Casa de Saúde da Idanha reab.cog. 